\title{
Predictors of prognosis in patients with temporal lobe epilepsy after anterior temporal lobectomy
}

\author{
ZHENXING SUN $^{1 *}$, HUANCONG ZUO ${ }^{1}$, DAN YUAN ${ }^{2}$, YAXING SUN $^{3}$, \\ $\mathrm{KAI} \mathrm{ZHANG}^{4 *}$, ZHIQIANG CUI ${ }^{1}$ and $\mathrm{JIN} \mathrm{WANG}^{5}$ \\ ${ }^{1}$ Department of Neurosurgery, Yuquan Hospital, Tsinghua University, Beijing 100084; ${ }^{2}$ Department of Neurology, \\ The Luhe Teaching Hospital, Capital Medical University, Beijing 101149; ${ }^{3}$ Department of Psychiatry, \\ The Second Municipal Hospital of Zaozhuang, Zaozhuang, Shandong 277100; ${ }^{4}$ Department of Neurosurgery, \\ Tiantan Hospital, Capital Medical University, Beijing 101149; ${ }^{5}$ Department of Neurosurgery, \\ Changgung Hospital, Tsinghua University Medical Center, Beijing 100084, P.R. China
}

Received August 26, 2014; Accepted July 13, 2015

DOI: $10.3892 /$ etm.2015.2753

\begin{abstract}
The aim of this study was to evaluate the predictive value of prognostic factors for the surgical outcome of patients with mesial temporal lobe epilepsy (MTLE) using Engel seizure classification. The clinical data of 121 patients with MTLE who underwent anterior temporal lobectomy (ATL) and received a 1-year minimum follow-up were collected between January 2005 and December 2008. Patients were divided into seizure and seizure-free groups according to the Engel seizure classification. Univariate analysis and multivariate logistic regression analysis were used to analyze the potential predictive and prognostic factors, including medical history, clinical features of seizures, magnetic resonance imaging (MRI) and video-electroencephalogram (EEG) monitoring results. Univariate analysis indicated no statistically significant differences in gender, age at seizure onset, age at surgery, history of traumatic brain injury, perinatal anoxia, intracranial infection, family history of seizure, auras or site of surgery between the two groups; however, significant differences were detected in pre-surgical seizure duration, history of febrile seizures, seizure types, MRI and video-EEG results. Multivariate logistic regression analysis demonstrated that a pre-surgical seizure duration of $<10$ years, history of positive febrile seizures, simple complex partial seizure, positive MRI results and unilateral local video-EEG spikes may be considered as predictors of a good prognosis. These results indicate that remission may be achieved in patients with MTLE via
\end{abstract}

Correspondence to: Dr Jin Wang, Department of Neurosurgery, Changgung Hospital, Tsinghua University Medical Center, 168 Litang Road, Beijing 100084, P.R. China

E-mail:wja01068@btch.edu.cn

*Contributed equally

Key words: anterior temporal lobectomy, mesial temporal lobe epilepsy, predictor, prognosis the collection of accurate clinical information and adequate pre-surgical evaluation.

\section{Introduction}

Mesial temporal lobe epilepsy (MTLE) is the most common type of medically refractory epilepsy syndrome in adolescents and adults. MTLE usually presents at the inner aspect of the temporal lobe, and typically affects the hippocampus, parahippocampal gyrus and amygdala $(1,2)$. The pathophysiological substrate of this disease is usually hippocampal sclerosis (HS) (3). However, certain cases of MTLE present without the typical changes of mesial temporal sclerosis or other abnormalities on magnetic resonance imaging (MRI) scans (MRI-negative), and the seizure outcome is poor (4). Previous studies have demonstrated that surgery is a more effective therapeutic approach for epilepsy compared with anti-epileptic drugs (AEDs) in the majority of patients with MTLE (5-7). For this reason, certain scholars consider surgery to be the first choice of treatment for MTLE. Anterior temporal lobectomy (ATL) is the most commonly used surgical technique for treating MTLE. McIntosh et al (6) provided a systematic review summarizing 126 articles published between 1991 and 2001, and demonstrated that $60-70 \%$ of patients with MTLE were free of seizures following ATL surgery. However, the remaining $\sim 30 \%$ of patients continue to experience seizures to a certain extent, the cause of which remains unclear. The primary goal of epilepsy surgery is to achieve the long-term prevention of seizures. However, numerous studies have reported that the long-term prognosis is relatively poor compared with the short-term prognosis (6-15). The causes for long-term worsening of the surgical outcome are also unknown and the predictive factors of short-term and long-term prognosis may differ.

Therefore, it is crucial to investigate the predictive indicators of long-term outcome in patients with MTLE, which may improve general understanding of the causes of surgical failure. However, few studies have evaluated the influence of pre-surgical factors including medical history, clinical features of seizures, MRI and video-electroencephalography (EEG) 
monitoring results on the efficacy of epilepsy surgery, particularly on the long-term prevention of seizures. Accordingly, it is necessary to understand how to predict the prognosis from pre-surgical factors and select patients for surgery in order to improve the seizure-free rate in patients with MTLE. Therefore, in the present study, the clinical data of 121 patients with MTLE who underwent an ATL was analyzed, with at least 1 year of follow-up. Patients were allocated into different groups according to Engel seizure classification in order to evaluate the surgical results in terms of seizure outcomes, and to further analyze the potential value of predictive factors associated with long-term efficacy.

\section{Subjects and methods}

Subjects and inclusion criteria. A total of 162 patients with medically refractory temporal epilepsy syndrome that underwent ATL in Tiantan Hospital (Beijing, China) were recruited between January 2005 and December 2008. The inclusion criteria were as follows: i) All patients presented the clinical characteristics of MTLE for $>1$ year and had adequate trial of at $\geq 3$ first-line antiepileptic drugs for $>2$ years, but continued to suffer from seizures; ii) continuous non-invasive 32-channel video scalp EEG monitoring (including sphenoidal electrodes to determine ictal and interictal focal activity for $\geq 7$ days) supported the characteristics of MTLE; iii) MRI (1.5T scanners) and/or histopathological findings were characteristic of HS. The histopathological findings associated with HS were neuronal loss and glial proliferation in the hippocampal subfields CA1 and CA3 or in the dentate gyrus (16-18). The MRI findings associated with HS were decreased hippocampal volume secondary to neuronal loss, and increased hippocampal T2 signal secondary to gliosis (16-18). This study was supported by the ethics committee of Tsinghua University (Beijing, China). Informed consent was obtained from the patients for the use of their data.

Pre-surgical evaluation. The pre-surgical evaluation program was as follows: i) Medical histories included perinatal anoxia, family history of seizure, febrile seizures, intracranial infection and traumatic brain injury; ii) clinical data included gender, pre-surgical duration, auras, age at seizure onset, duration of seizure, types of seizure, seizure frequency per month prior to surgery, location of surgery and age at surgery; iii) diagnostic examination included MRI and video-EEG via scalp/sphenoidal electrodes, single photon emission computed tomography or positron emission tomography or magnetoencephalography of relevant areas of patients.

Surgical procedure. Following an ipsilateral frontotemporal craniotomy, all patients underwent cerebral cortex and deep EEG monitoring. The surgical approach adopted ATL during which the anterior $4-5 \mathrm{~cm}$ of the temporal tip for the dominant hemisphere and 5-6 cm for the non-dominant one were resected, as were the mesial structures including the areas of amygdala, hippocampus and parahippocampal gyrus. The extent of resection was adjusted on the basis of intraoperative electrocorticogram (ECoG) monitoring. All surgeries were performed by the same neurosurgeon and no patients underwent a second surgery.
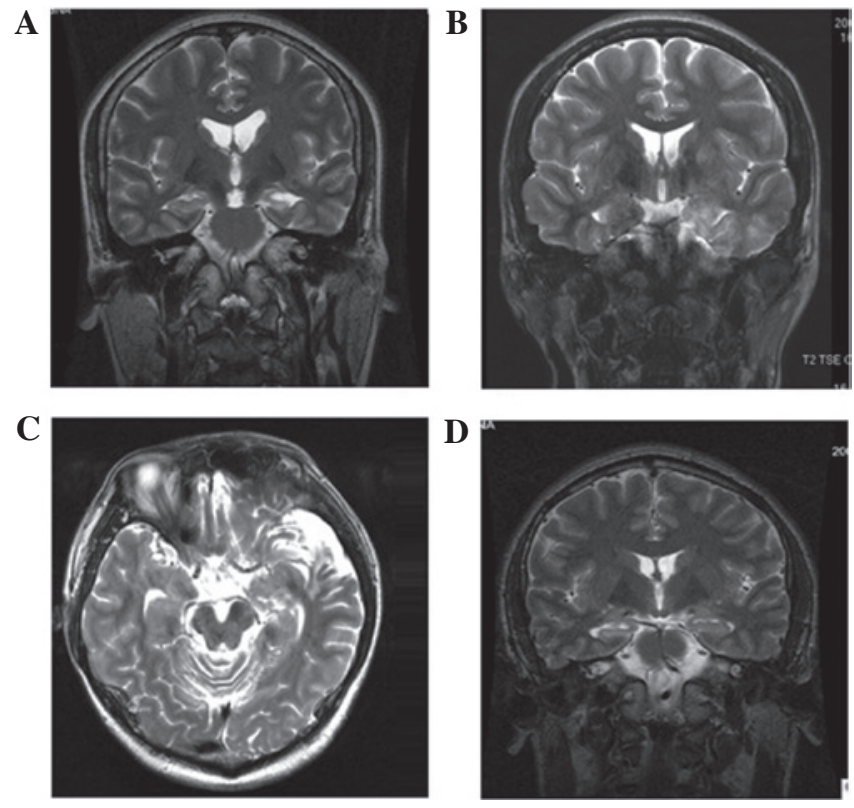

Figure 1. Representative micrographs of magnetic resonance images showing hippocampal sclerosis or normal hippocampus. (A) Left hippocampus appeared atrophied and hyperintense on the T2-weighted images, which was diagnosed as hippocampal sclerosis (HS). (B) Cortical dysplasia in the left temporal lobe with left HS. (C) Cortical atrophy in the left temporal lobe with HS. (D) The temporal horn in the right lateral ventricle was expanded with a normal hippocampus.

Follow-up. Postoperative follow-up data regarding seizure outcomes, including clinical auras, seizure frequency and duration of seizure, were obtained via standardized telephone interviews performed by one of the authors. Patients were assigned to outcome classes in relation to seizure control based on the Engel seizure classification, which was as follows: Class I, free of disabling seizures; class II, rare disabling seizures, 'almost seizure-free'; class III, worthwhile improvement; and class IV, no worthwhile improvement.

Data collection and statistical analysis. The pre-surgical seizure duration was grouped as $<10$ years and $\geq 10$ years. According to the international classification of epileptic seizures (19), seizure types were divided into simple complex partial seizures (CPS), frequent CPS and frequent generalized tonic-clonic seizures (GTCS) based on whether the CPS was accompanied by GTCS or not. The MRI data were classed as positive and negative according to whether HS was present or not (Fig. 1). Patients were further classified as exhibiting unilateral local lobe, unilateral multi-lobe or bilateral lobe seizures, based on the characteristics of the video-EEG.

Regarding seizure outcome, patients were divided into two categories: Seizure-free group (Engel classification, I) and seizure group (Engel classification, II-IV). Patients with non-disabling auras, but without seizures, were considered to be seizure-free. Seizure freedom was defined as those patients that were seizure-free from the day of surgery to the last outcome assessment, or were seizure-free for $>2$-years post surgery.

An independent sample t-test or Rank sum test was used to analyze the quantitative variables: Age at seizure onset, pre-surgical duration, duration of seizure, seizure frequency 
Table I. Patient characteristics.

\begin{tabular}{lc}
\hline Parameter & Value \\
\hline Gender, M/F & $63 / 58$ \\
Follow-up period, years & \\
Mean & $3.33 \pm 0.89$ \\
Range & $1-5$
\end{tabular}

Age at seizure onset, years

Mean

Range

$16.35 \pm 12.29$

4-34

Age at surgery, years

Mean

Range

$27.55 \pm 11.58$

$5-54$

Pre-surgical seizure duration, years

Mean

Range

History associated with seizure, $\mathrm{n}(\%)$

Febrile seizures, $\mathrm{n}(\%)$

Traumatic brain injury, $\mathrm{n}(\%)$

Intracranial infection, $\mathrm{n}(\%)$

Perinatal anoxia, $\mathrm{n}(\%)$

Family history of seizures, $\mathrm{n}(\%)$

Surgery location, $\mathrm{n}(\%)$

Left side

Right side

Clinical auras, $\mathrm{n}(\%)$

Gastric gas

Palpitation

Headache

Fear

Déjà-vu

Euphoria

Sadness

Automatism, n (\%)

Oroalimentary

Gestural

Verbal

Seizure types, $\mathrm{n}(\%)$

Single CPS

Frequent CPS

Frequent GTCS

Video-EEG results, $\mathrm{n}(\%)$

Unilateral lobe

Unilateral multi-lobe

Bilateral lobes

$10.90 \pm 7.50$

$83(68.6)$

$63(52.1)$

19 (15.7)

7 (5.8)

$12(9.9)$

$2(1.7)$

$61(50.4)$

60 (49.6)

$57(47.1)$

$11(9.1)$

$13(10.7)$

7 (5.8)

$17(14.0)$

$6(5.0)$

$1(0.8)$

$2(1.7)$

$53(43.8)$

$22(18.2)$

$25(20.7)$

$6(5.0)$

$30(24.8)$

$72(59.5)$

$19(15.7)$

$90(74.4)$

17 (14.0)

14 (11.6)

Evidence of HS, n (\%)

MRI

$101(83.5)$

Histopathology per month prior to surgery and age at surgery. A Pearson's $\chi^{2}$, continuity correction or Fisher's exact test was used to analyze the following qualitative variables: Gender, presence of auras, history of perinatal anoxia, family history of seizure, febrile seizures, intracranial infection and traumatic brain injury, location of surgery and MRI data. Row x Column (RxC) contingency table $\chi^{2}$ and subdivided $\mathrm{RxC}$ table independence tests were used for the analysis of seizure types and distributing range of spikes in the video-EEG. Backward stepwise multiple logistic regression analysis was used to assess the possible predicting factors of prognosis. Using SPSS software, version 16.0 (SPSS, Inc., Chicago, IL, USA) P $<0.017$ was considered to indicate a statistically significant difference in the subdividing $\mathrm{RxC}$ table tests, and $\mathrm{P}<0.025$ was considered to indicate a statistically significant difference for the other statistical methods.

\section{Results}

Patient characteristics. Among the total of 162 patients with ATL, 123 (75.9\%) exhibited MTLE/HS, 14 (8.6\%) gliomas, $4(2.5 \%)$ heterotopic gray matter, $5(3.1 \%)$ cavernous malformation, 8 (4.9\%) cerebral dysgenetic lesions and 8 (4.9\%) arachnoid cysts. A total of 121 patients $(98.4 \%, 63$ men and 58 women) among the 123 patients with MTLE/HS were adequately followed-up. The mean follow-up period was $3.33 \pm 0.89$ years (range, $1-5$ years). The mean patient ages at seizure onset and at surgery were $16.35 \pm 12.29$ years (range, 4-34 years) and 27.55 \pm 11.58 years (range, 5-54 years), respectively. The mean patient pre-surgical seizure period was $10.90 \pm 7.50$ years (range, 2-35 years). Among the 121 patients that received follow-up, 83 cases possessed a history relating to seizures, including 63 cases with febrile seizures (including 43 simple febrile seizures without secondary diseases, 7 cases secondary to intracranial infection and 13 cases secondary to traumatic brain injury), 19 cases with traumatic brain injury, 7 cases with intracranial infection, 12 cases with perinatal anoxia and 2 cases with a family history of seizures. The numbers of patients that underwent ATL on the left and right side were $61(50.4 \%)$ and 60 (49.6\%), respectively (Table I).

Among the 57 patients with auras, 11 cases presented with adverse rising of gastric gas, 13 cases had palpitations, 7 cases suffered from headache, 17 cases experienced fear, 6 cases had déjà-vu, 1 case experienced euphoria and 2 cases exhibited sadness, as observed by the doctor. In addition, 53/121 patients (43.8\%) had automatism, including 22 cases of oroalimentary automatism, 25 cases of gestural automatism and 6 cases of verbal automatism. With regard to seizures, there were 30 patients $(24.8 \%)$ with CPS, 72 patients $(59.5 \%)$ with frequent CPS and 19 patients (15.7\%) with frequent GTCS. The results of the video-EEG showed that in 90 patients (74.4\%) the unilateral local lobe was involved in the seizure onset, 17 patients (14.0\%) had unilateral multi-lobe involvement and 14 patients (11.6\%) had bilateral lobe involvement. Among the 121 patients, 101 cases $(83.5 \%)$ presented with indications of HS under MRI examination, and 115 cases (93.4\%) had a diagnosis of HS confirmed by histopathological analysis (the remaining 6 patients were not available for histopathological examination but were confirmed to have HS using MRI). Therefore, all patients received a confirmed diagnosis of HS

M/F, male/female; CPS, complex partial seizures; GCTS, generalized tonic-clonic seizures; EEG, electroencephalogram; HS, hippocampal sclerosis; MRI, magnetic resonance imaging. 
Table II. Year-by-year seizures by Engel class.

\begin{tabular}{lrr}
\hline & \multicolumn{2}{c}{ Patients, n (\%) } \\
\cline { 2 - 3 } Follow-up & \multicolumn{1}{c}{ Class I } & Classes II-IV \\
\hline 1 year & $87 / 121(71.9)$ & $34 / 121(28.1)$ \\
2 years & $83 / 116(71.6)$ & $33 / 116(28.4)$ \\
3 years & $69 / 91(75.8)$ & $22 / 91(24.2)$ \\
4 years & $41 / 52(78.8)$ & $11 / 52(21.2)$ \\
5 years & $11 / 16(68.8)$ & $5 / 16(31.2)$ \\
\hline
\end{tabular}

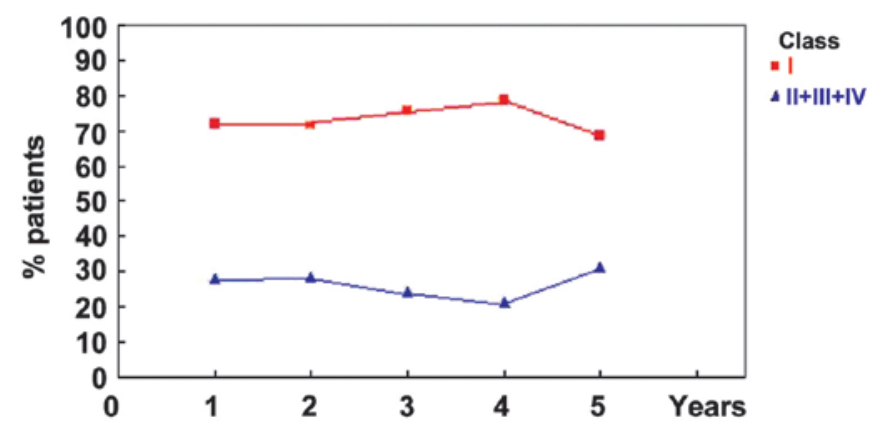

Figure 2. Patient outcome in the Engel class I (seizure-free) group was improved compared with that of the class II-IV (seizure) group. Year-by-year seizure outcomes were analyzed based on Engel's classification during the 5-year follow-up period.

using MRI or histopathological analysis, including 20 patients in whom MRI did not reveal HS, and 95 patients (78.5\%) in whom HS was confirmed by MRI and histopathological results (Table I).

Engel classification. In total, 87 patients (71.9\%) received an Engel classification of class I and formed the seizure-free group. In addition, 3 (2.5\%), 18 (14.9\%) and 13 patients (10.7\%) were categorized as Engel class II, III and IV, respectively, and were grouped together as the seizure group. Furthermore, 87/121 (71.9\%), 83/116 (71.6\%), 69/91 (75.8\%), 41/52 (78.8\%) and $11 / 16(68.8 \%)$ patients satisfied the criteria of Engel Class I at years 1-5 of follow-up, respectively. Year-by-year seizure outcomes based on Engel's classification are presented in Table II and Fig. 2.

Identification of variables distinguishing between seizure and seizure-free groups. Univariate analysis indicated no statistically significant difference between the seizure and seizure-free groups in gender, age at onset of seizures, age at surgery, history of traumatic brain injury, perinatal anoxia, intracranial infection, family history of seizures, presence of auras and site of surgery. By contrast, significant differences were detected in pre-surgical duration $<10$ years, history of febrile seizures, seizure type, MRI and video-EEG results between the two groups. The outcomes of analyses between the seizure-free and seizure groups are presented in Tables III-V.

The Rank sum test indicated no statistically significant difference between the seizure and seizure-free groups with regard to duration of seizures and seizure frequency prior to surgery. However, the $\mathrm{RxC} \chi^{2}$ test revealed a statistically significant difference in video-EEG results and seizure types between the two groups. Furthermore, the subdividing RxC table test showed that the seizure outcomes for unilateral local lobe involvement differed in comparison with those for multiple lobe involvement (including unilateral multi-lobes and bilateral lobes) and that seizure outcomes involving CPS differed from those of frequent CPS and frequent GTCS (Table V).

On the basis of a backward stepwise multiple logistic regression analysis, the presence of a number of pre-surgical risk factors, including pre-surgical seizure duration, history of febrile seizures, seizure types, MRI and video-EEG results were associated with poor prognosis. However, other factors, including gender, presence of auras, age at seizure onset, age at surgery, duration of seizures, seizure frequency per month prior to surgery, history of perinatal anoxia, intracranial infection or traumatic brain injury, and a family history of seizure were not associated with prognosis (Table VI).

\section{Discussion}

MTLE is the most common type of temporal lobe epilepsy (TLE), and surgery is the most effective treatment available at present for this condition $(1,2,5,6,20)$. Since the causes of surgical failure are not understood, epilepsy surgery is underutilized. Surgical approaches include ATL, amygdalohippocampectomy and lesionectomy/corticectomy. Various alternative methods of resecting MTLE have been discussed since ATL was first developed by Falconer and Taylor (21). However, at present ATL remains the predominant surgical option for MTLE (5-7) and the seizure outcomes following ATL have been extensively studied $(6-8,18)$. Prognostic factors for the long-term surgical outcomes of MTLE include early postoperative seizures $(22,23)$, duration of seizures $(24,25)$, age at surgery (24-26), history of febrile seizures $(11,27)$, concordant interictal epileptiform abnormalities $(20,28,29)$ and GTCS or the presence of status epilepticus prior to surgery $(7,10,29,30)$. However, there are few systematic studies regarding the prediction of long-term seizure-free outcome following ATL. In addition, the previous studies regarding the surgical outcomes of MTLE are conflicting. The discrepancies in these predictive factors may be associated with the differences in the pathological substrates used and methodological problems of the study population $(6,31)$.

The aim of the present study was to identify prognostic factors that are able to predict the surgical outcome in patients with MTLE at 1-5 years after ATL, according to the well-established Engel classification system. The results of 121 patients that had undergone a range of pre-surgical evaluations, including medical history, clinical presentation, MRI and video-EEG observations, are reported. Among these patients, $71.9 \%$ with MTLE became seizure free and the remaining $28.1 \%$ patients continued to exhibit seizure symptoms at the first follow-up period, which was consistent with the results of previous studies $(6,7,26)$. Seizure relief rates have ranged between 67 and $84 \%$ for the surgical treatment of MTLE in recent studies $(6,7,26)$ with a median rate of $\sim 70 \%$ (6). This outcome may further support the use of early ATL for the treatment of MTLE. However, in the present study, 
Table III. Analysis of identifying variables between seizure-free and seizure groups.

\begin{tabular}{lccc}
\hline Quantitative variables & Seizure-free $(\mathrm{n}=87)$ & Seizure $(\mathrm{n}=34)$ & \\
\hline t-test & & & \\
Age at surgery (years) & $26.97 \pm 11.70$ & $29.21 \pm 11.81$ & 0.347 \\
Age at onset of seizure (years) & $16.44 \pm 12.33$ & $16.12 \pm 12.37$ & 0.897 \\
Pre-surgical duration (years) & $10.39 \pm 7.56$ & $11.89 \pm 7.61$ & 0.329 \\
Rank sum test & & $146.67 \pm 218.20$ & 0.908 \\
Duration of seizures (sec) & $136.67 \pm 170.44$ & $40.94 \pm 83.81$ & 0.935 \\
Seizure frequency (n/month) & $23.37 \pm 46.34$ &
\end{tabular}

Quantitative results are expressed as the mean \pm standard deviation.

Table IV. Analysis of identifying variables between seizure-free and seizure groups.

\begin{tabular}{lccc}
\hline Qualitative variables & Seizure-free $(\mathrm{n}=87)$ & Seizure $(\mathrm{n}=34)$ & P-value \\
\hline Pre-surgical duration $<10$ years & 41 & 23 & 0.042 \\
Age at surgery <14 years & 14 & 20 & 0.301 \\
Site of surgery (left temporal) & 41 & 15 & 0.247 \\
Gender (male) & 48 & 24 & 0.274 \\
MRI (positive) & 77 & 14 & 0.017 \\
Presence of auras & 43 & 16 & 0.414 \\
Past histories & 67 & 5 & 0.001 \\
Febrile seizures & 58 & 6 & 0.001 \\
Traumatic brain injury & 13 & 3 & 0.713 \\
Perinatal anoxia & 9 & 1 & 1.000 \\
Family history of seizure & 1 & 1 & 0.000 \\
Intracranial infection & 6 & 36 \\
\hline
\end{tabular}

Pearson's $\chi^{2}$ continuity correction of Fisher's exact test was used for statistical analysis. MRI, magnetic resonance imaging.

Table V. Analysis of identifying variables between seizure-free and seizure groups.

\begin{tabular}{|c|c|c|c|}
\hline Qualitative variables & Seizure-free $(n=87)$ & Seizure $(n=34)$ & P-value \\
\hline Video-EEG results & & & 0.003 \\
\hline Bilateral lobes & 7 & 7 & \\
\hline Unilateral multi-lobes & 8 & 9 & \\
\hline Unilateral local lobe & 72 & 18 & \\
\hline Seizure types & & & 0.001 \\
\hline Frequent GTCS & 7 & 12 & \\
\hline Frequent CPS & 57 & 15 & \\
\hline Simple CPS & 23 & 7 & \\
\hline
\end{tabular}

RxC contingency tables and $\chi^{2}$ tests were used for statistical analysis. EEG, electroencephalogram; GTCS, generalized tonic-clonic seizures; CPS, complex partial seizures.

the seizure-free rate of the patients had decreased by 5 years after surgery and the seizure-free rates $>5$ years after surgery are unknown. Therefore, the therapeutic efficacy of surgery after 5 years cannot be predicted. Furthermore, certain predictors of the long-term surgical outcomes following ALT for MTLE may differ from the variables that predict short-term 
Table VI. Backward stepwise multiple logistic regression.

Engel classification

\begin{tabular}{|c|c|c|}
\hline \multirow[b]{2}{*}{ Parameter } & \multicolumn{2}{|c|}{ Engel classification } \\
\hline & $\begin{array}{l}\text { Regression } \\
\text { coefficient }\end{array}$ & P-value \\
\hline $\begin{array}{l}\text { Pre-surgical seizure } \\
\text { duration }<10 \text { years }\end{array}$ & 1.100 & 0.019 \\
\hline History of febrile seizures & 1.755 & 0.002 \\
\hline Seizure types & 1.232 & 0.017 \\
\hline Video-EEG findings & 1.321 & 0.012 \\
\hline MRI (positive) & 1.441 & 0.005 \\
\hline Constant & -7.500 & 0.001 \\
\hline
\end{tabular}

EEG, electroencephalogram; MRI, magnetic resonance imaging.

outcome. In the present study, univariate and multivariate analyses indicated that factors including pre-surgical seizure duration, history of febrile convulsions, seizure types, MRI and video-EEG results exhibited statistically significant differences between the seizure and seizure-free group, and these factors were associated with the surgical outcomes of the patients.

In the present study, of the 64 patients with a seizure duration $<10$ years, $41(64 \%)$ were in the seizure-free group and $23(36 \%)$ were in the seizure-free group. Of the 57 patients with a pre-surgical seizure duration of $\geq 10$ years, 46 patients $(80.7 \%)$ belonged to the seizure-free group and 11 patients $(19.3 \%)$ were in the seizure group. The Pearson's $\chi^{2}$ test indicated a statistically significant difference in seizure outcome between the two groups $(\mathrm{P}=0.042)$. Backward stepwise logistic regression further demonstrated that a pre-surgical duration of $<10$ years predicted a seizure-free 5 -year outcome. In addition, previous studies have demonstrated that patients with a longer pre-surgical seizure duration exhibit a poor outcome, and are more likely to exhibit large epileptogenic zones and more diffuse lesions, which are difficult to eradicate with surgery $(32,33)$. On the basis of these observations, it was hypothesized for the purposes of the present study that epilepsy duration may be a key predictive factor of long-term surgical outcome.

In addition, it was observed that a history of febrile seizures was a predictor for surgical outcome following ALT according to backward logistic analysis. Among the 121 patients were followed up, 63 cases (52.1\%) had a history of febrile seizures while 58 (47.9\%) had no history of febrile seizures, which is consistent with a previous study by Pavlidou et al (34), in which patients with a history of febrile seizures comprised $\sim 40 \%$ of TLE cases, particularly in children aged $<4$ years (34). Among the 63 patients with a history of febrile seizures, 58 (92.1\%) were classified as seizure-free, which was significantly different from the rate among the 58 patients with no history of febrile seizures (29/58, 50.0\%). This observation indicates that the structural and functional anatomy of the brain was altered, becoming more susceptible to intractability following febrile seizures $(11,27,34)$.

On the basis of univariate and multivariate analysis, seizure types were found to be associated with long-term surgical outcome evaluated $\leq 5$ years following surgery. A $\chi^{2}$ test revealed statistically significant differences in seizure outcome between simple CPS and frequent CPS or frequent GTCS. Furthermore, it was observed that 19 patients (15.7\%) exhibited frequent GTCS, including 7 patients (36.8\%) that had a good outcome and 12 patients $(63.2 \%)$ with a poor outcome. These results indicate that GTCS was associated with a worse outcome. Theoretically, patients with CPS would be expected to exhibit an improved outcome compared with patients with frequent CPS, based on previous studies $(13,22)$. By contrast, it was observed that $57 / 72$ patients (79.2\%) with frequent CPS were seizure-free, while $23 / 30$ patients $(76.7 \%$ ) with simple CPS exhibited improved seizure control. This result may be due to epileptogenic zones in patients with GTCS being difficult to localize and resect, resulting in the postoperative formation of a residual epileptogenic area.

With respect to video-EEG results, $72 / 90$ patients $(80.0 \%)$ with unilateral local lobe involvement, $8 / 17$ patients $(47.1 \%)$ with unilateral multi-lobe involvement and $7 / 14$ patients $(50.0 \%)$ with bilateral lobe involvement exhibited a seizure-free outcome. Furthermore, positive video-EEG results were a predictor of surgical outcome, which indicates that patients with unilateral lobe involvement may expect an improved outcome compared with patients with bilateral lobe involvement. Therefore, the present study detected a significant predictive contribution of video-EEG data, particularly in patients with MTLE or with normal MRI results, which is similar to the findings of previous studies $(7,10,14,32)$. However, in other studies, discrepancies between video-EEG and MRI results indicated a poor surgical outcome, which may be due to the existence of bilateral HS or multi-lobe foci $(16,35)$.

Finally, 77/101 patients (76.2\%) with positive MRI were seizure-free and 24/101 patients (23.8\%) were in the seizure group, indicating that MRI may be useful for the pre-surgical evaluation of epilepsy patients. On the basis of multiple logistic regression analysis, it was determined that the presence of MRI abnormalities was highly predictive of long-term patient outcome. Furthermore, various MRI results, particularly the presence of mesial temporal sclerosis, were identified as predictive of surgical outcomes, which is consistent with previous studies that reported a poor outcome for patients that exhibited no signs of MTLE under MRI $(33,36-39)$. In addition, discrepancies between MRI and histopathological results regarding MTLE may be due to the fact that current MRI protocols only provide accurate information in patients with severe bilateral HS or with a significant difference in the bilateral hippocampus. Therefore, the majority of patients with mild unilateral HS and severe contralateral HS are diagnosed with unilateral HS, while patients with mild bilateral HS are considered to be normal $(31,35)$. Although these patients underwent preoperative MRI examinations, for the majority of patients MRI results were not available for each year during the follow-up period. Thus, the structural changes occurring in the brain following surgery are unknown, particularly with respect to the residual hippocampal tissue following ATL, which was a common cause of recurrent seizures, and further studies are required to investigate this in the future.

In the present study, it was demonstrated that the majority of patients with MTLE may be treated effectively with an ATL. A number of pre-surgical factors, including pre-surgical 
seizure duration, history of febrile convulsion, seizure type, MRI and video-EEG results may be used as predictors of surgical outcomes. Thus, postoperative remission may be achieved in patients with MTLE via the accurate collection of patient information and adequate pre-surgical evaluation. Further studies are required that include a larger group of patients with a longer follow-up period, in order to analyze the rate of late seizure recurrence after an initial seizure-free period. In addition, year-by-year MRI examination and comparisons between MRI and video-EEG results may aid in ascertaining surgical outcomes.

\section{Acknowledgements}

The authors thank Wenhan $\mathrm{Hu}$ and Ning Chen for collecting the data from the archive, as well as Chao Zhang for technical help.

\section{References}

1. Engel J Jr: The timing of surgical intervention for mesial temporal lobe epilepsy: A plan for a randomized clinical trial. Arch Neurol 56: 1338-1341, 1999.

2. Wieser HG; ILAE commission on neurosurgery of epilepsy: ILAE commission report. Mesial temporal lobe epilepsy with hippocampal sclerosis. Epilepsia 45: 695-714, 2004.

3. Engel J Jr: Mesial temporal lobe epilepsy: What have we learned? Neuroscientist 7: 340-352, 2001.

4. Wieser HG and Hane A: Antiepileptic drug treatment in seizure-free mesial temporal lobe epilepsy patients with hippocampal sclerosis following selective amygdalohippocampectomy. Seizure 13: 534-536, 2004

5. Berkovic SF, McIntosh AM and Kalnins RM: Preoperative MRI predicts outcome of temporal lobectomy: An actuarial analysis. Neurology 45: 1358-1363, 1995.

6. McIntosh AM, Wilson SJ and Berkovic SF: Seizure outcome after temporal lobectomy: current research practice and findings. Epilepsia 42: 1288-1307, 2001.

7. Radhakrishnan K, So EL, Silbert PL, et al: Predictors of outcome of anterior temporal lobectomy for intractable epilepsy: A multivariate study. Neurology 51: 465-471, 1998.

8. Blume WT, Desai HB, Girvin JP, McLachlan RS and Lemieux JF: Effectiveness of temporal lobectomy measured by yearly follow-up and multivariate analysis. J Epilepsy 7: 203-214, 1994.

9. Guldvog B, Løyning Y, Hauglie-Hanssen E, Flood S and Bjørnas H: Predictive factors for success in surgical treatment for partial epilepsy: A multivariate analysis. Epilepsia 35: 566-578, 1994.

10. Hennessy MJ, Elwes RD, Rabe-Hesketh S, Binnie CD and Polkey CD: Prognostic factors in the surgical treatment of medically intractable epilepsy associated with mesial temporal sclerosis. Acta Neurol Scand 103: 344-350, 2001.

11. Janszky J, Schulz R and Ebner A: Clinical features and surgical outcome of medial temporal lobe epilepsy with a history of complex febrile convulsions. Epilepsy Res 55: 1-8, 2003.

12. Specht U, May T, Schulz R, et al: Cerebellar atrophy and prognosis after temporal lobe resection. J Neurol Neurosurg Psychiatry 62: 501-506, 1997.

13. Semah F, Picot MC, Adam C, et al: Is the underlying cause of epilepsy a major prognostic factor for recurrence? Neurology 51 : 1256-1262, 1998.

14. Schulz R, Lüder HO, Hoppe M, Tuxhorn I, May T and Ebner A: Interictal EEG and ictal scalp EEG propagation are highly predictive of surgical outcome in mesial temporal lobe epilepsy. Epilepsia 41: 564-570, 2000.

15. Spencer SS, Berg AT, Vickrey BG, et al: Initial outcomes in the multicenter study of epilepsy surgery. Neurology 61: 1680-1685, 2003.

16. Bote RP, Blázquez-Llorca L, Fernández-Gil MA, Alonso-Nanclares L, Muñoz A and De Felipe J: Hippocampal sclerosis: Histopathology substrate and magnetic resonance imaging. Semin Ultrasound CT MR 29: 2-14, 2008.
17. Camacho DLA and Castillo MMR: Imaging of temporal lobe epilepsy. Semin Ultrasound CT and MRI 28: 424-436, 2007.

18. Tanriverdi T, Olivier A, Poulin N, Andermann F and Dubeau F: Long-term seizure outcome after mesial temporal lobe epilepsy surgery: Corticalamygdalohippocampectomy versus selective amygdalohippocampectomy. J Neurosurg 108: 517-524, 2008.

19. Bancaud J, Henriksen O, Rubio-Donnadieu F, Seino M, Dreifuss FE and Penry JK; Commission on the Classification and Terminology of the International League Against Epilepsy: Proposal for revised clinical and electroencephalographic classification of epileptic seizures. Epilepsia 22: 489-501, 1981.

20. Wiebe S, Blume WT, Girvin JP and Eliasziw M: Effectiveness and efficiency of surgery for temporal lobe epilepsy study group. A randomized, controlled trial of surgery for temporal-lobe epilepsy. N Engl J Med 345: 311-318, 2001.

21. Falconer MA and Taylor DC: Surgical treatment of drug-resistant epilepsy due to mesial temporal sclerosis: Etiology and significance. Arch Neurol 19: 353-361, 1968.

22. McIntosh AM, Kalnins RM, Michell LA and Berkovic SF: Early seizures after temporal lobectomy predict subsequent seizure recurrence. Ann Neurol 57: 283-288, 2005.

23. Tezera FI, Akalanb N, Oguzc KK, Karabulut E, Dericioglu N, Ciger A and Saygi S: Predictive factors for postoperative outcome in temporal lobe epilepsy according to two different classifications. Seizure 17: 549-560, 2008

24. Clusmann H, Schramm J, Kral T, et al: Prognostic factors and outcome after different types of resection for temporal lobe epilepsy. J Neurosurg 97: 1131-1141, 2002.

25. Zentner J, Hufnagel A, Wolf HK, et al: Surgical treatment of temporal lobe epilepsy: Clinical, radiological and histopathological findings in 178 patients. J Neurol Neurosurg Psychiatry 58: 666-673, 1995.

26. Jeong SW, Lee SK, Kim KK, Kim H, Kim JY and Chung CK: Prognostic factors in anterior temporal lobe resections for mesial temporal lobe epilepsy: Multivariate analysis. Epilepsia 40: 1735-1739, 1999.

27. Behrens E, Zentner J, van Roost D, Hufnagel A, Elger CE and Schramm J: Subdural and depth electrodes in the presurgical evaluation of epilepsy. Acta Neurochir (Wien) 128: 84-87, 1994.

28. Foldvary N, Nashold B, Mascha E, et al: Seizure outcome after temporal lobectomy for temporal lobe epilepsy: A Kaplan-Meier survival analysis. Neurology 54: 630-634, 2000.

29. Ho SS, Berkovic SF, McKay WJ, Kalnins RM and Bladin PF Temporal lobe epilepsy subtypes: Differential patterns of cerebral perfusion on ictal SPECT. Epilepsia 37: 788-795, 1996.

30. Clusmann H, Kral T, Fackeldey E, et al: Lesional mesial temporal lobe epilepsy and limited resections: Prognostic factors and outcome. J Neurol Neurosurg Psychiatry 75: 1589-1596, 2004.

31. Schramm J: Temporal lobe epilepsy surgery and the quest for optimal extent of resection: A review. Epilepsia 49: 1296-1307, 2008.

32. Holmes MD, Born DE, Kutsy RL, Wilensky AJ, Ojemann GA and Ojemann LM: Outcome after surgery in patients with refractory temporal lobe epilepsy and normal MRI. Seizure 9: 407-411, 2000.

33. Sperling MR, Saykin AJ, Glosser G, et al: Predictors of outcome after anterior temporal lobectomy: The intracarotid amobarbital test. Neurology 44: 2325-2330, 1994.

34. Pavlidou E, Tzitiridou M, Kontopoulos E and Panteliadis CP: Which factors determine febrile seizure recurrence? A prospective study. Brain Dev 30: 7-13, 2008.

35. Vinton AB, Carne R, Hicks RJ, et al: The extent of resection of FDG-PET hypometabolism relates to outcome of temporal lobectomy. Brain 130: 548-560, 2007.

36. Antel SB, Li LM, Cendes F, et al: Predicting surgical outcome in temporal lobe epilepsy patients using MRI and MRSI. Neurology 58: 1505-1512, 2002.

37. Clusmann H: Predictors, procedures and perspective for temporal lobe epilepsy surgery. Semin Ultrasound CT MR 29: 60-70, 2008.

38. Gilliam F, Bowling S, Bilir E, et al: Association of combined MRI, interictal EEG, and ictal EEG results with outcome and pathology after temporal lobectomy. Epilepsia 38: 1315-1320, 1997.

39. Pillai JJ, Williams HT and Faro S: Functional imaging in temporal lobe epilepsy. Semin Ultrasound CT MR 28: 437-450, 2007. 